\title{
Editorial
}

\section{Reconfigurable Electromagnetics through Metamaterials}

\author{
Giacomo Oliveri, ${ }^{1}$ Douglas Werner, ${ }^{2}$ Filiberto Bilotti, ${ }^{3}$ and Christophe Craeye ${ }^{4}$ \\ ${ }^{1}$ ELEDIA Research Center@DISI, University of Trento, 38123 Trento, Italy \\ ${ }^{2}$ Department of Electrical Engineering, The Pennsylvania State University, University Park, PA 16802, USA \\ ${ }^{3}$ Department of Engineering, Roma Tre University, 00146 Rome, Italy \\ ${ }^{4}$ Ecole Polytechnique de Louvain, Université catholique de Louvain, 1348 Louvain-la-Neuve, Belgium
}

Correspondence should be addressed to Giacomo Oliveri; giacomo.oliveri@disi.unitn.it

Received 17 April 2014; Accepted 17 April 2014; Published 15 June 2014

Copyright ( 2014 Giacomo Oliveri et al. This is an open access article distributed under the Creative Commons Attribution License, which permits unrestricted use, distribution, and reproduction in any medium, provided the original work is properly cited.

In recent years there has been a growing interest in the development of artificial materials, in particular in metamaterials, engineered in order to exhibit electromagnetic properties that do not occur in nature. The high degree of enthusiasm over electromagnetic metamaterials stems from their ability to manipulate and channel electromagnetic waves in unprecedented ways, which is derived from their subwavelength structure/granularity, rather than being based solely on their constitutive materials. These features of electromagnetic metamaterials promise a path toward completely new devices with properties and functionality not possible with currently available technologies. This is specifically true whenever reconfigurability is of interest (e.g., for nonconventional and transformative applications in telecommunications, medicine, and security). Indeed, due to their intrinsic structure, electromagnetic metamaterials represent a key tool to implement the "reconfigurable electromagnetics" paradigm that is to design and fabricate devices which can be controlled through a change of the physical properties of their constitutive materials at a subwavelength scale.

In this framework, the special issue is aimed at reviewing the most recent advances of metamaterials as an enabling technology for the design and realization of reconfigurable devices at $\mathrm{RF}$ and $\mathrm{THz} / \mathrm{optical}$ frequencies, and it includes 7 papers representing the state-of-the-art work being carried out in this topic area by some of the top university research labs around the world. This collection provides a comprehensive overview of some of the most interesting advancements in reconfigurable metamaterials from the technological, methodological, and experimental viewpoint, and it highlights some of the potentialities and future trends in this research area.

More in detail, the paper by J. P. Turpin et al. presents a comprehensive review of the developments of reconfigurable and tunable metamaterials as well as of the applications of such technology. A survey of several different tuning methods, including circuit-enabled tuning, geometrical tuning, and material tuning, is presented, and their applicability over different frequency bands (from RF to optical frequencies) is discussed. A set of applications is also reported that comprises tunable filters and antennas, devices with adjustable scattering parameters, tunable GRIN lenses, and antennas with reconfigurable propagation features. Additionally, some of the open challenges towards their applicability in practical devices are discussed.

A review of some of the recent contributions to materials where the dynamic control is enabled by micro-electromechanical systems (MEMS) technology is presented in the paper by T. Debogovic and J. Perruisseau-Carrier. More specifically, efficient reconfigurable phase shifters and leakywave antennas (LWA) based on reconfigurable composite right/left-handed transmission lines are illustrated. Moreover, very low loss metasurface designs with reconfigurable reflection properties are discussed along with their application in reflectarrays and partially reflective surfaces. Fabrication and experimental validation of the presented devices in the $\mathrm{X}$ - and $\mathrm{Ku}$-bands are also reported.

N. J. Smith et al. present a conformal wideband metamaterial array achieving a 10:1 continuous bandwidth. To 
accomplish this goal, a wideband Marchand-type balun spanning the bandwidth from $280 \mathrm{MHz}$ to $2800 \mathrm{MHz}$ was designed and measured; its reconfiguration capabilities are obtained by means of circuit changes in the balanced feed integrated with the wideband metamaterial array. The potentialities of the metamaterial array's reconfiguration are demonstrated through five example bandpass and bandrejection responses.

The paper by J. Naqui and F. Martin is concerned with the exploitation of microstrip transmission lines loaded with stepped impedance resonators (SIRs) etched on top of a signal strip to achieve mechanically reconfigurable metamaterials. More in detail, it is shown that the notch frequency and depth of the transmission line can be mechanically controlled by acting on the symmetry of the transmission line itself. Such a property is then exploited for the implementation of sensors and electronic barcodes.

An innovative surface wave antenna is presented in the paper by Q. Bai et al. More in detail, the novel low-profile switchable antenna is based on band gap materials that can support both surface waves and normal modes of communication. The techniques for generating a dual mode switchable antenna are reported, and the performance of the antenna is investigated. The surface wave communication mode is also assessed by using an EBG to couple antennas/sensors together around the body.

The paper by A. Vallecchi et al. presents single and dual polarisation operation arrays based on reconfigurable bistate metasurfaces composed of interwoven spiral arrays with embedded PIN diodes. More specifically, the array response between transmission and reflection modes at the specified frequencies is changed by means of the PIN diodes, which are controlled through the DC bias supplied by the spiral conductors forming the metasurface. A set of simulation results is reported to illustrate the good isolation between transmission and reflection states, as well as the excellent angular and polarisation stability of the proposed active metasurfaces over a large fractional bandwidth.

Finally, the paper by C. Argyropoulos et al. discusses several tunable and reconfigurable designs of linear and nonlinear plasmonic and hyperbolic metamaterials. More in detail, rich scattering features of multilayered composite nanoparticles are demonstrated; they include exotic scattering signatures that combine multiple dipolar Fano resonances and electromagnetic induced transparency (EIT) features. Moreover, the exploitation of nonlinear hyperbolic metamaterial designs is addressed to realize tunable positive-tonegative refraction at the same frequency. Several devices are envisioned, based on the proposed tunable metamaterials, including ultrafast reconfigurable imaging systems, tunable sensors, novel nanotag designs, and efficient alloptical switches and memories.

\section{Acknowledgments}

We would like to thank the International Journal of Antennas and Propagation for the opportunity to serve as Guest Editors of this special issue. Moreover, we would like to thank all of the authors for their patience with us and with the review process. We hope that you will find this special issue on the subject of reconfigurable electromagnetics interesting. The work reported in these manuscripts demonstrates that such a topic represents an extremely active interdisciplinary research field with the promise of significant scientific and industrial opportunities.

Giacomo Oliveri

Douglas Werner

Filiberto Bilotti

Christophe Craeye 

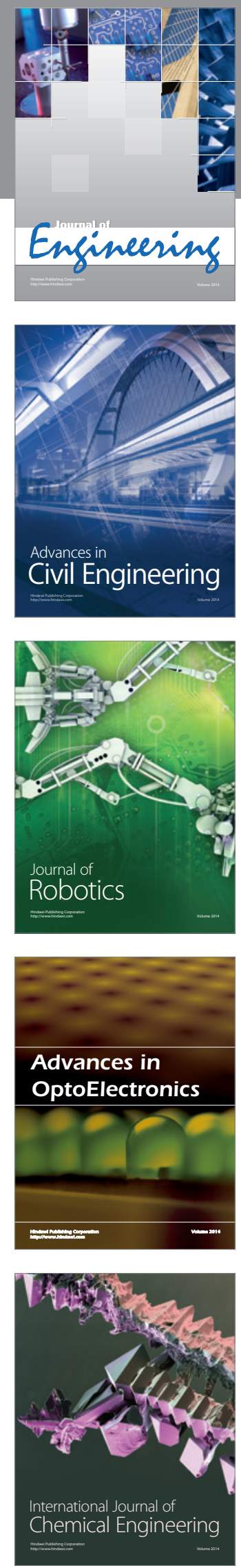

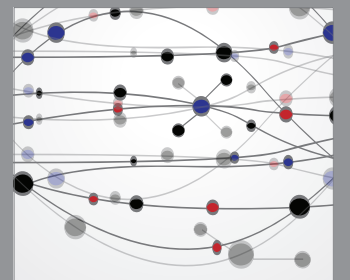

The Scientific World Journal
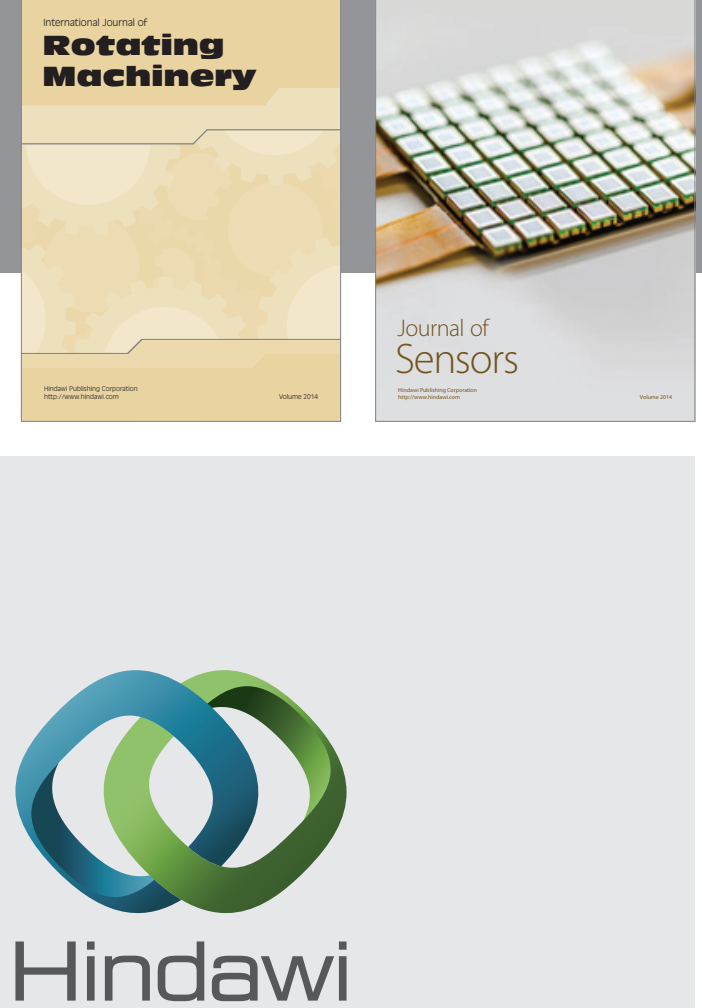

Submit your manuscripts at http://www.hindawi.com
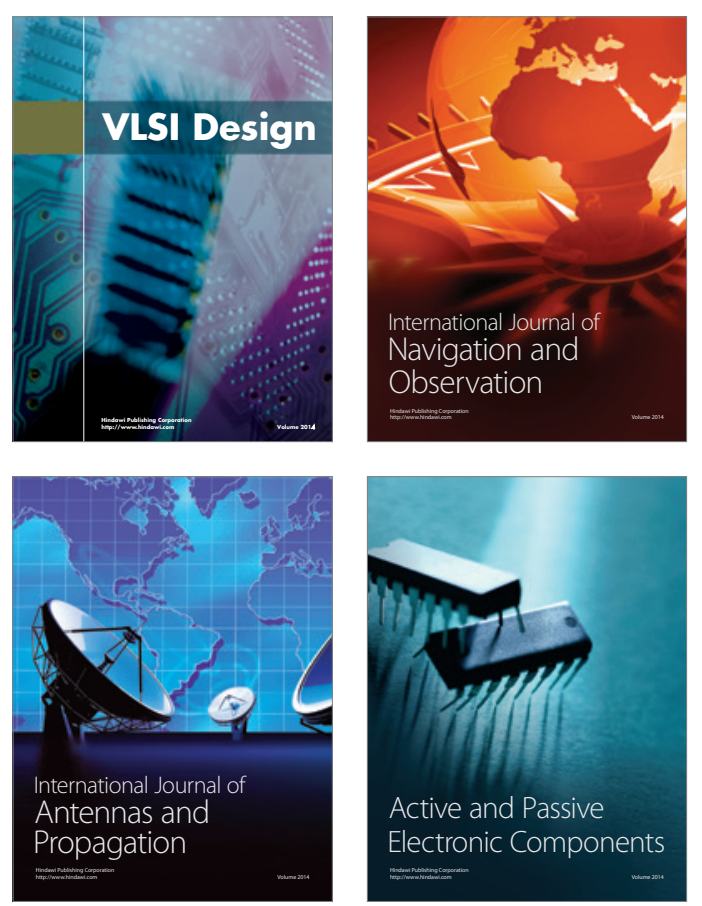
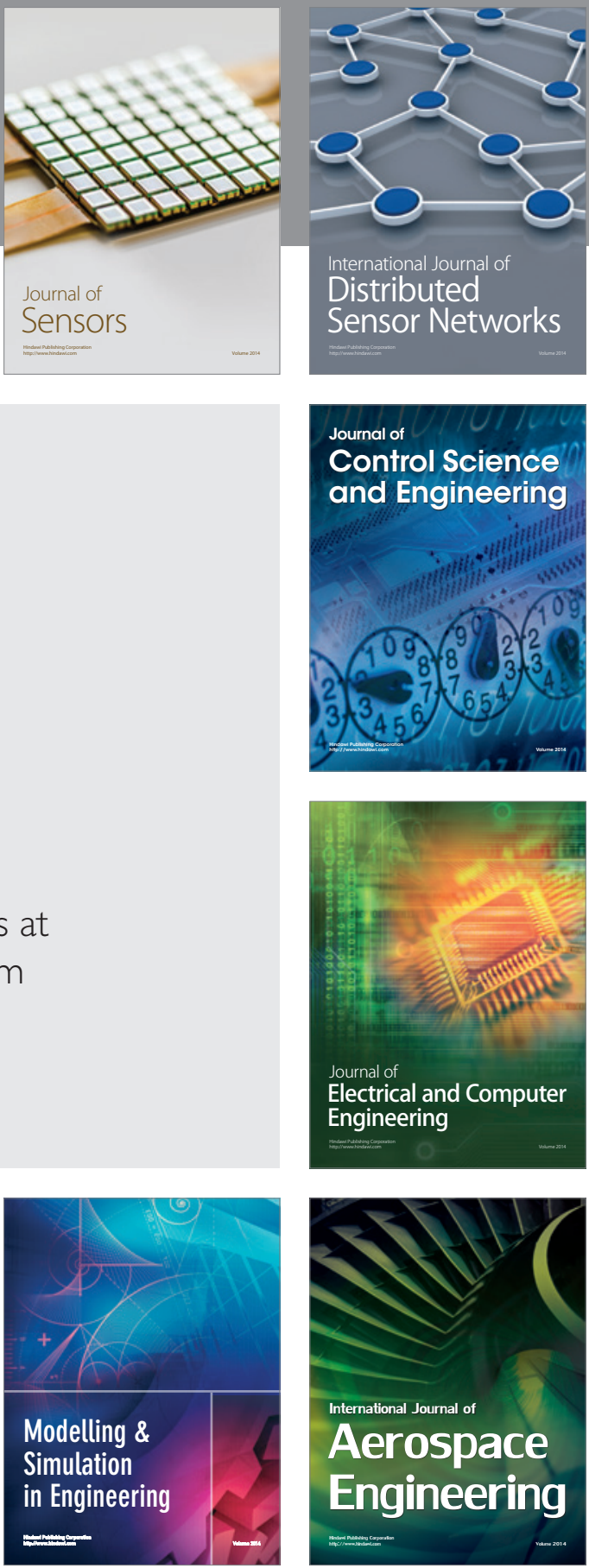

Journal of

Control Science

and Engineering
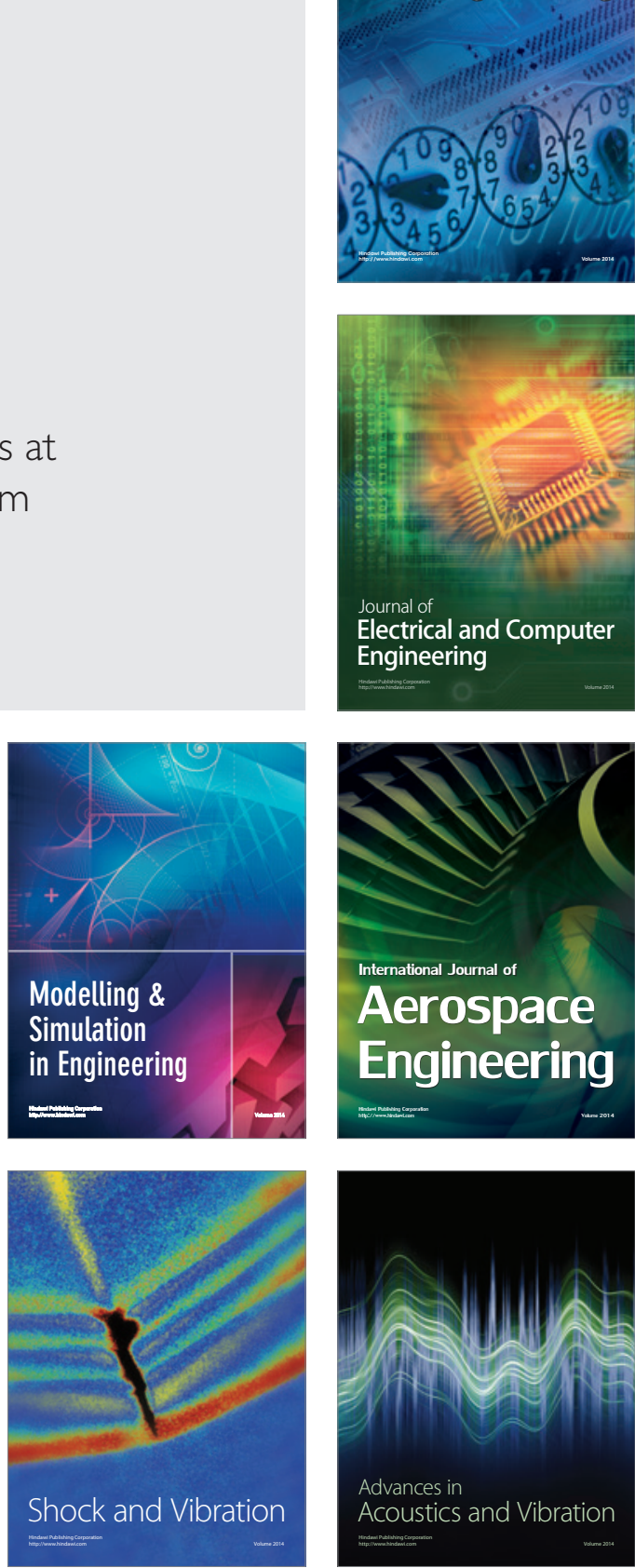